\title{
A new design for sampling with adaptive sample plots
}

\author{
Haijun Yang • Christoph Kleinn • \\ Lutz Fehrmann • Shouzheng Tang • \\ Steen Magnussen
}

Received: 25 April 2009 / Revised: 17 November 2009 / Published online: 11 December 2009

(C) The Author(s) 2009. This article is published with open access at Springerlink.com

\begin{abstract}
Adaptive cluster sampling (ACS) is a sampling technique for sampling rare and geographically clustered populations. Aiming to enhance the practicability of ACS while maintaining some of its major characteristics, an adaptive sample plot design is introduced in this study which facilitates field work compared to "standard" ACS. The plot design is based on a conditional plot expansion: a larger plot (by a pre-defined plot size factor) is installed at a sample point instead of the smaller initial plot if a pre-defined condition is fulfilled. This study provides insight to the statistical performance of the proposed adaptive plot design. A design-unbiased estimator is presented and used on six artificial and one real tree position maps to estimate density (number of objects per ha). The performance in terms of coefficient of variation is compared to the non-adaptive alternative without a conditional expansion of plot size.
\end{abstract}

H. Yang $\cdot$ C. Kleinn $(\varangle) \cdot$ L. Fehrmann

Forest Inventory and Remote Sensing, Georg-August-Universität Göttingen,

Büsgenweg 5, 37077 Göttingen, Germany

e-mail: ckleinn@gwdg.de

H. Yang

e-mail: hyang@gwdg.de

L. Fehrmann

e-mail: lfehrma@gwdg.de

S. Tang

Institute of Forest Resources Information Techniques, Chinese Academy of Forestry,

P.O. Box 9, 100091 Beijing, People's Republic of China

e-mail: stang@caf.ac.cn

S. Magnussen

Pacific Forestry Centre, Canadian Forest Service, Natural Resources Canada,

506 West Burnside Road, Victoria, BC V8Z 1M5, Canada

e-mail: steen.magnussen@nrcan.gc.ca 
The adaptive plot design was superior in all cases but the improvement depends on (1) the structure of the sampled population, (2) the plot size factor and (3) the critical value (the minimum number of objects triggering an expansion). For some spatial arrangements the improvement is relatively small. The adaptive design may be particularly attractive for sampling in rare and compactly clustered populations with an appropriately chosen plot size factor.

Keywords Forest inventory · Adaptive cluster sampling · Plot design · Conditional plot expansion · Inclusion zone approach

\section{Introduction}

Forest inventories and ecological surveys are tools of data collection to support decision making in forest management and conservation. Forest inventories on a statistical basis have developed over a period of about a century. Their scope is continuously widening, especially during the past decades. Many techniques of sampling designs and response designs with proven track records in forestry are also successfully being employed in ecological surveys. Both share a series of common challenges of which one is sampling for "rare events" (Thompson 2004). Rare events are target objects such as plant species that occur in low density. Frequently, the abundance of rare species is of a particular interest for conservation and biodiversity monitoring. Accurate information about rare objects is difficult to achieve with conventional inventory designs.

When sampling for rare events, a conventional sampling approach with a predefined number of $n$ sample plots of fixed area (in the terminology of ecological surveys: quadrats) leads to high relative standard errors, owing to a large proportion of empty plots. This is particularly pronounced, when target objects occur in groups and the standard error is determined from a majority of empty plots and a few plots with possibly high numbers of target objects.

Many rare plant species appears to grow in groups of individual plants, including palms, shrubs, trees and medicinal and aromatic plants. For such situations, Thompson (1990) suggested a technique referred to as adaptive cluster sampling (ACS): sample points are selected according to a sampling design, for example, random or systematic sampling, and at each sample location a square plot, called initial plot, is laid out. If on an initial plot a specific condition is satisfied-for example: "at least one target object found"- then the adjacent square plots are also tallied, where-in the original work of Thompson (1990) — the population is imagined to be completely tessellated into square plots. If one of these adjacent square plots meets the condition, their neighboring plots are tallied as well, and so on. That is, the initial plot is step by step enlarged by adding more and more plots and becomes an irregularly shaped cluster of sub-plots. Eventually the cluster will contain the entire group of target objects intersected by the initial plot. Thus, the cluster plot adapts in size and shape to the group of target objects encountered at a sample location. If no target object is found on an initial plot then the cluster consists only of the initial plot. Further details of the classic ACS and proposed estimators can be found in Thompson (1990). 
ACS can be used with any sampling design. Extensive research has confirmed this for simple random sampling, systematic sampling, stratified sampling (Thompson 1991), inverse sampling (Christman and Lan 2001), two stage sampling (Muttlak and Khan 2002), double sampling (Félix-Medina and Thompson 2004), and for designs with or without replacement of clusters (Dryver 1999; Salehi and Seber 1997). The focus is usually on the comparison and improvement of estimators (Dryver 2003; Dryver and Thompson 2005; Félix-Medina 2003; Salehi 1999, 2003; Salehi and Seber 2002). ACS applications include surveys of trees (Acharya et al. 2000; Roesch 1993), terrestrial herpetofauna (Noon et al. 2006), low-abundance plants (Phillipi 2005), medicinal plants (Acworth 1999), other non-timber forest products (Bih 2007; Wong 2001), and fish (Hanselman et al. 2003).

Adaptive cluster plots can be superior to conventional response designs when a population of interest is rare and geographically clustered and the triggering condition is appropriately defined (Acharya et al. 2000; Christman 1997; Magnussen et al. 2005; Phillipi 2005; Smith et al. 2003; Talvitie et al. 2006). However, to our knowledge ACS has not yet been used on a routine basis in field surveys as they pertain to, for example, biodiversity monitoring and forest inventory. A lack of field applications may be due to the fact that the final size of adaptive cluster plots is difficult to predict. With large groups of target objects or groups connected (at the scale of initial plots) by individual elements, the adaptive process could expand the plot to an extent where they become prohibitively expensive. Some authors have recommended a stopping rule (Brown and Manly 1998; Chao and Thompson 1999; Lo et al. 1997) that applies when a predefined number of subplots in an adaptive cluster-plot is reached.

In this article, we propose an alternative to ACS that is both adaptive and practical. Our example has trees as objects and the number of trees (density) as the target variable for estimation. Yet the technique applies to any kind of target object and any target variable(s). A generally applicable estimator is developed for the adaptive plot design and its performance is assessed using both artificial and real datasets.

\section{An adaptive plot approach with conditional plot expansion}

To overcome practical issues of differently sized, gradually growing and possibly very large adaptive clusters of sub-plots with the conventional adaptive cluster design strategy, we suggest a more basic approach with only two pre-defined plot-sizes: (1) a common initial plot installed at each sample point, and (2) a larger (adapted) but fixed sized plot installed at sample points where a pre-defined condition is met. For example, a standard initial plot size of $500 \mathrm{~m}^{2}$ is installed at each sample point; and if the condition "at least one tree on the initial plot" is fulfilled, then the $500 \mathrm{~m}^{2}$ plot is expanded to a $1,500 \mathrm{~m}^{2}$ plot. Figure 1 illustrates the expansion principle.

With a conditional plot expansion rather than a non-adaptive design, more trees are likely to be recorded. In comparison to standard ACS, its field implementation is easier as the same simple predefined plot expansion rule holds for all initial plots and the adaptation process needs not to be done in a time-consuming and error-prone sequential fashion. In contrast to conventional ACS a fixed conditional plot expansion 


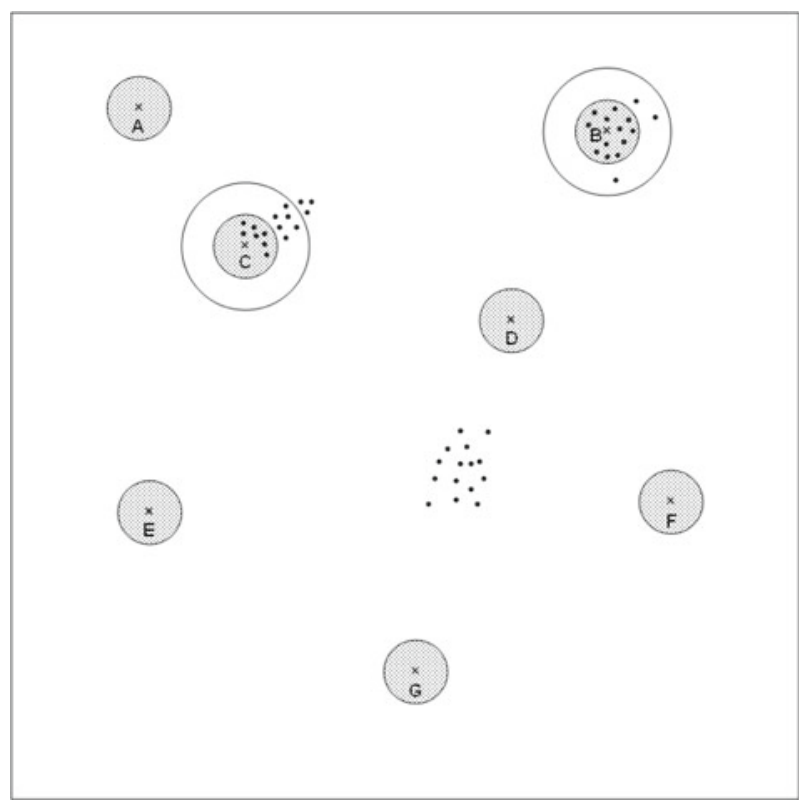

Fig. 1 An example of the adaptive plot design: an initial sample plot of a defined size (gray shaded) is established at each sample point (crosses $A$ to $G$ ). When the condition: the initial plot contains at least one tree (black dot) - is met, the plot is expanded to a fixed larger size (shown for two initial plots at sample point $B$ and $C$ ). The final plot size is the same for all expanded plots

rule does not, however, ensure a capture of all trees in an isolated group intercepted by an initial plot.

In this study both the initial and the expanded plots are circular. Circular plots are common in forest inventory because of practicality. However, the principle of conditional plot expansion is also applicable to other shapes.

The plot expansion is determined by the requirement to be met and by the geometry of the expansion. In our example, the condition for an expansion is stated in terms of the number of target trees found in an initial (non-expanded) plot. The number needed to trigger an expansion is termed the "critical value" $(\mathrm{CrV})$. The plot size factor (PSF) defines how much larger the expanded plot area is in relation to the initial plot: a plot size factor of two means a doubling of the plot area.

\section{Estimator}

The standard technique used with fixed area plots to extrapolate the per-plot observations to per-hectare values cannot be applied to adaptive plot designs because it requires equal inclusion probability for all trees at a given sample point. In the proposed design the inclusion probability is obviously not constant: a tree with other trees nearby has a higher probability of inclusion than an isolated tree, because the selection of a neighboring tree in an initial plot may lead to the selection of that particular tree in the expanded plot. 


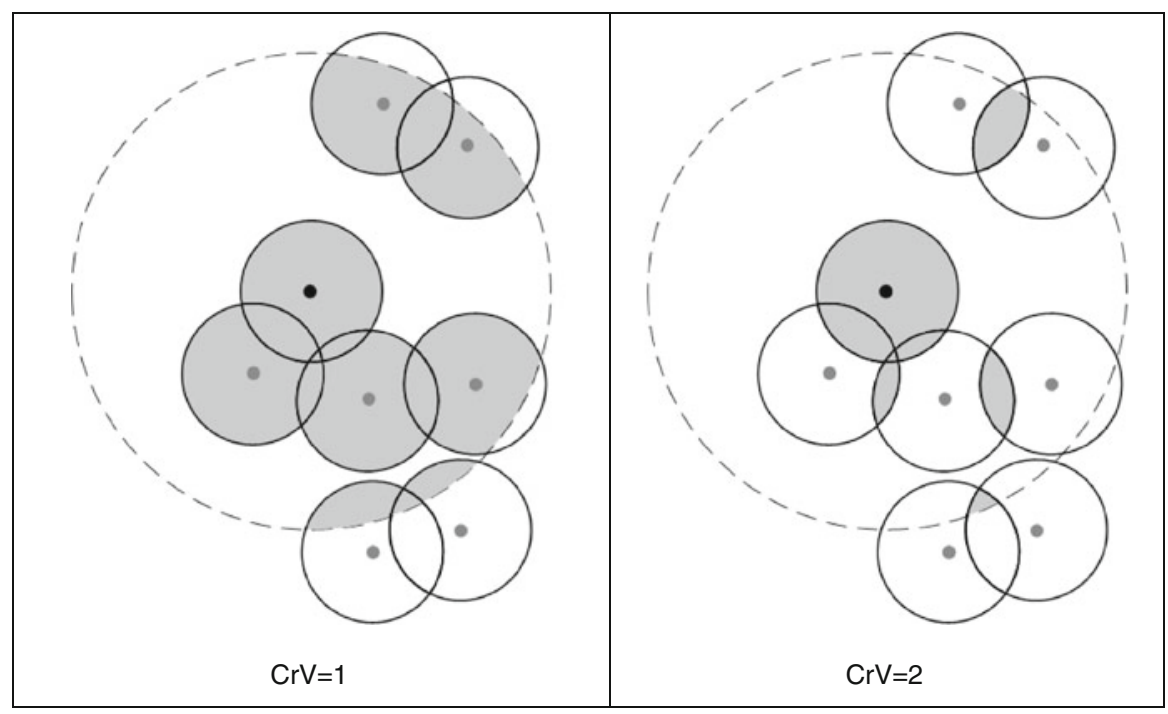

Fig. 2 Two examples of inclusion zones for the center tree. For explanation of the construction principles see text

Our estimation design adopts the infinite population paradigm which considers the dimensionless points in the area of interest as sampling elements (Eriksson 1995). The paradigm is described in, for example, Gregoire and Valentine (2008) and Mandallaz (2008). Construction of design-unbiased estimators for various plot designs is straightforward under this paradigm (for example fixed count sampling, Kleinn and Vilčko 2006), because it analytically determines the inclusion probability for each selected sample tree. Once inclusion probabilities are known the Horvitz-Thompson estimator framework applies directly. A probabilistic sampling design for selection of the initial sample points is a prerequisite; here we restrict ourselves to simple random sampling.

The inclusion probability of a particular tree can be derived from its inclusion zone constructed geometrically as the surrounding area that leads to its inclusion if a sample point falls within it. The area of its inclusion zone divided by the domain area of the population is a geometrical measure of that particular tree's inclusion probability.

The basic question becomes how to construct an inclusion zones and determine its area for a given sample tree. In our case, the zone varies with the factors that define the plot design (initial plot size, PSF, and CrV) and the number and spatial arrangement of trees. Figure 2 schematically illustrates an example for a center tree and for a fixed PSF but two CrVs (left: "at least one tree in the initial plot" and right: "at least two trees in the initial plot"). All sample points falling inside the gray filled inclusion zone leads to an inclusion of the center tree in a sample. The gray filled area is a direct measure of its inclusion probability. For a CrV of 1 (Fig. 2, left), the center tree (target tree) is included if it is either directly inside an initial plot or if at least one of its neighboring trees are within an initial plot which would trigger an expansion that subsequently includes the focus tree. The two inclusion paths for a tree translate into the shown inclusion zones CrVs of 1 and 2 (Fig. 2). 
To determine the inclusion zone of a tree, only that particular tree and its neighbors whose initial-plot based inclusion zones overlap the big circle centered at it are relevant. The big circle is of the same size as an expanded plot. For $\mathrm{CrV}=1$ the inclusion zone of the centered target tree is then constructed from the intersection between the big circle (dashed line) and the union of the initial-plot based inclusion zones of all relevant trees. For $\mathrm{CrV}=2$ the inclusion zone of the center tree can be depicted from the intersection of the union of the initial-plot based joint inclusion zones of all relevant neighbors and the initial-plot based inclusion zone of the center tree with the big circle.

Obviously, as can be seen from Fig. 2, the determination of an actual inclusion zone for a particular tree requires the coordinates of all trees up to a distance that equals the sum of radii of the expanded plot and the initial plot. In consequence, a maximal circular area of $(2 \sqrt{\mathrm{PSF}}+1)^{2}$ times the size of the initial plot around a sample point has to be observed for coordinates when a plot is expanded. At sample point locations where the initial plot contains target objects but the predefined condition is not satisfied (their number is smaller than the defined $\mathrm{CrV})$, at most a circular area of $(\sqrt{\mathrm{PSF}}+2)^{2}$ times the initial plot size have to be checked. The collection of coordinates relative to the sample point is indispensable unless more straightforward approaches are identified to determine the inclusion probability or appropriate proxies, and incurs additional efforts.

Once the inclusion zones of all sampled trees have been analytically determined and their areas calculated, the Horvitz-Thompson estimator applies for the estimation of the total of any obtained tree characteristic.

Following largely the notation in Valentine et al. (2001), the estimate for the total $\dot{T}_{k}$ for the inventory region of size $A_{T}$ derived from the observations at sample point $k$ is then:

$$
\dot{T}_{k}=\sum_{i=1}^{N} \frac{Y_{i}}{A_{i} / A_{T}} Z_{k i}
$$

with

$N=$ total number of trees in the inventory area,

$A_{i}=$ inclusion area of tree $i$,

$Y_{i}=$ observed value of the target variable at tree $i$,

$Z_{k i}=\left\{\begin{array}{l}1, \text { if sample point } k \text { falls into the inclusion zone of tree } i \\ 0, \text { otherwise. }\end{array}\right.$

From the individual observations as produced at each sample point, the estimators for the total $\hat{T}$ and its variance $\operatorname{var}(\hat{T})$ from $n$ sample points are, respectively,

$$
\hat{T}=\frac{\sum_{k=1}^{n} \dot{T}_{k}}{n} \text { and } \operatorname{vâr}(\hat{T})=\frac{\sum_{k=1}^{n}\left(\dot{T}_{k}-\hat{T}\right)^{2}}{n(n-1)},
$$

and this applies to totals of any tree variable. In the sampling simulation, estimating the number of stems per ha in the inventory regions is taken as an example. 


\section{Simulation study}

One real and six artificial populations were used to evaluate the statistical performance of the new adaptive plot design (Fig. 3). The real population consists of 268 individuals of the shrub species Tamarix ramosissim mapped on a rectangular study site of $500 \mathrm{~m} \times 100 \mathrm{~m}$. Data were collected from Inner Mongolia, China, in 2007. It is referred to as TR. The six artificial populations, referred to as SIM1 to SIM6, were generated from a variation of a Poisson cluster process (Diggle 2003). Each has the same spatial domain and number of individuals as TR - only the plant locations are varied.

In the six artificial populations, the number of clusters and the number of individuals per cluster were generated at random from two Poisson distributions with means of 3 and 90, respectively. In addition, the spatial extension of the clusters varied. The coordinates of each cluster center were generated by random coordinates. The position of a tree within a cluster is then determined relative to its cluster center by random azimuth and distance from a continuous uniform distribution with a location parameter 0 and a scale parameter $\lambda$. The $\lambda$ values used for the six artificial populations were $5,10,20,30$ and $100 \mathrm{~m}$ (with two different spatial arrangements) respectively. The resulting spatial distributions are shown in Fig. 3.

\section{Comparison of the performance of the plot designs}

To compare the performance of the proposed adaptive plot design it is necessary to know for each of the seven inventory regions the variance of the per-plot observations of the target variable for both the adaptive and the non-adaptive approach. As all points in the inventory area are potential sample plot centers, this population is infinite and the sought population characteristics are traditionally approximated by Monte-Carlo simulations of a large number of random samples. However, Roesch et al. (1993) introduced a direct approach to calculate these characteristics by finding the selection probabilities for each distinct set of sample trees as defined by the plot design, the so-called jigsaw-puzzle approach. This approach is illustrated in Fig. 4 and is being used in this study to calculate the true variance of density estimates.

We solved, with standard GIS-techniques, the jigsaw puzzle for the non-adaptive plot design and for the adaptive plot design for all possible levelcombinations of three factors (1) initial plot size (circular plot radius $R=10$ and $15 \mathrm{~m}$ ), (2) $\mathrm{CrV}(1,2,3)$ and (3) PSF $(1,2, \ldots, 8)$. From this tessellation, the selection probabilities of all possible sample plots are known and parametric values of the per-plot-observations can be directly calculated and need not to be approximated by Monte-Carlo simulation. The resulting inclusion probabilities for both the adaptive and the non-adaptive plots are design-unbiased and the populations are identical with respect to the parametric value ( $N=268$ ). Therefore, the statistical performance for any sample size can directly be compared by means of any measure of variability of sample plot observations. Here, we use the coefficients of variation.

The adaptive plot design demands more time in the field than fixed-area plot designs. A comparison of overall efficiency would require assumptions about the additional 
Fig. 3 Maps of the six artificial populations SIM1-SIM6 and the real population TR of mapped Tamarix ramosissim in Inner Mongolia, China (bottom). All maps have 268 trees as in the real population TR; the artificially generated populations SIM1 to SIM6 are arranged in three major clusters of different size characterized by the scale parameter $\lambda$ : $\operatorname{SIM} 1(\lambda=5 \mathrm{~m})$, SIM2 $(\lambda=10 \mathrm{~m})$, SIM3 $(\lambda=20 \mathrm{~m})$ and SIM4 $(\lambda=30 \mathrm{~m}), \operatorname{SIM} 5(\lambda=100 \mathrm{~m})$, SIM6 $(\lambda=100 \mathrm{~m})$
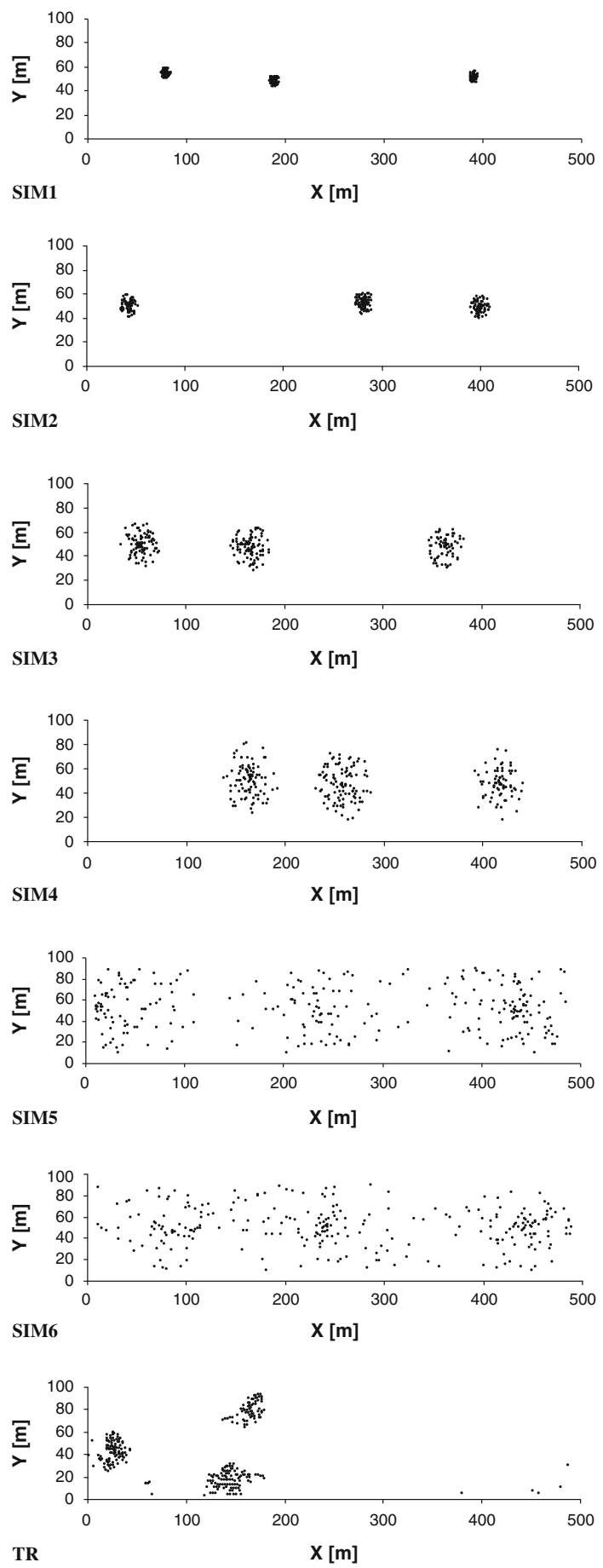


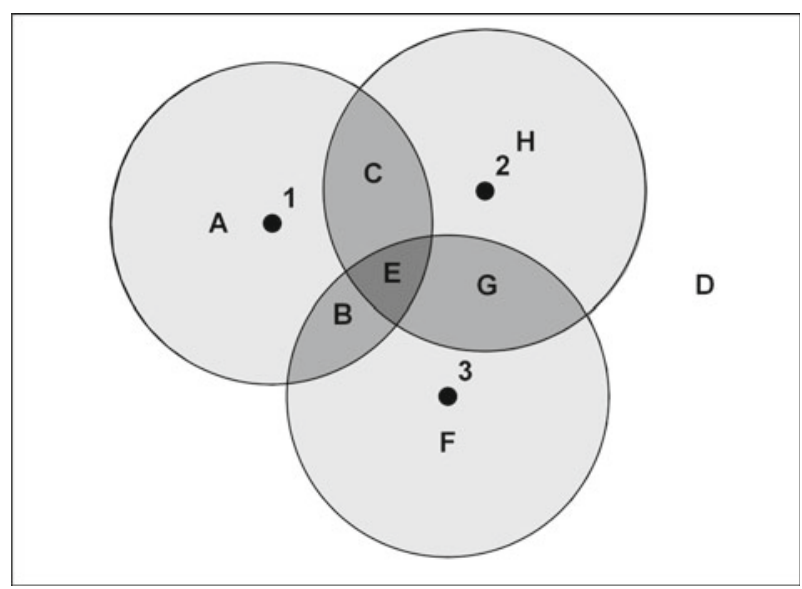

Fig. 4 Illustration of the jigsaw puzzle approach as introduced by Roesch (1993), applied to fixed area circular sample plots. Around each tree there is a circular inclusion zone. Inclusion zones of neighbouring trees can overlap and the resulting tesselation in non-overlapping pieces is akin to a "jigsaw puzzle" that covers the entire area without any gaps. All sample points within one specific "jigsaw" piece ( $A, B, C, D, E$, $F, G$ or $H$ ) lead to the selection of exactly the same set of trees. That is, the probability of any possible set of sample trees to be selected by simple random sampling with a given plot design is defined by the area of one particular piece of this jigsaw puzzle

field effort and related cost functions, which is beyond the scope of this study. Only statistical efficiency is considered.

\section{Results}

\subsection{Plot size factor and initial plot size}

The results for our choices of PSF are given in Fig. 5. General trends are: (1) a larger initial plot size produces less variability, as expected, (2) the adaptive plot design lowers the variability in density estimates for all PSFs $>1$ (3) the coefficients of variation $\mathrm{CV} \%$ decrease with increasing PSF but at diminishing rates of decline as PSF gets larger. This point of leveling-off occurs slightly earlier for the larger initial plot size $R=15 \mathrm{~m}$ for all maps. The PSF value beyond which the decline in CV\% is trivial (Fig. 5) was for population SIM1 about 2.5 and 2 for initial sample plot radius $10 \mathrm{~m}$ and $15 \mathrm{~m}$, respectively, about 5 and 3 for population SIM2, and about 7 and 5 for population SIM3 and about 8 for both initial plot sizes in SIM4. For the other study populations the point of level-off did not appear in the considered range of PSF. The differences in performance can be explained by the different size of clusters. In SIM1 a relatively small PSF-value can lead to capture of an entire cluster. In other populations a larger PSF is needed to accomplish the same (e.g. SIM4 and TR). However, in SIM5 and SIM6 where neighboring clusters may overlap, a very large PSF is needed to achieve a comparable reduction of $\mathrm{CV} \%$. 

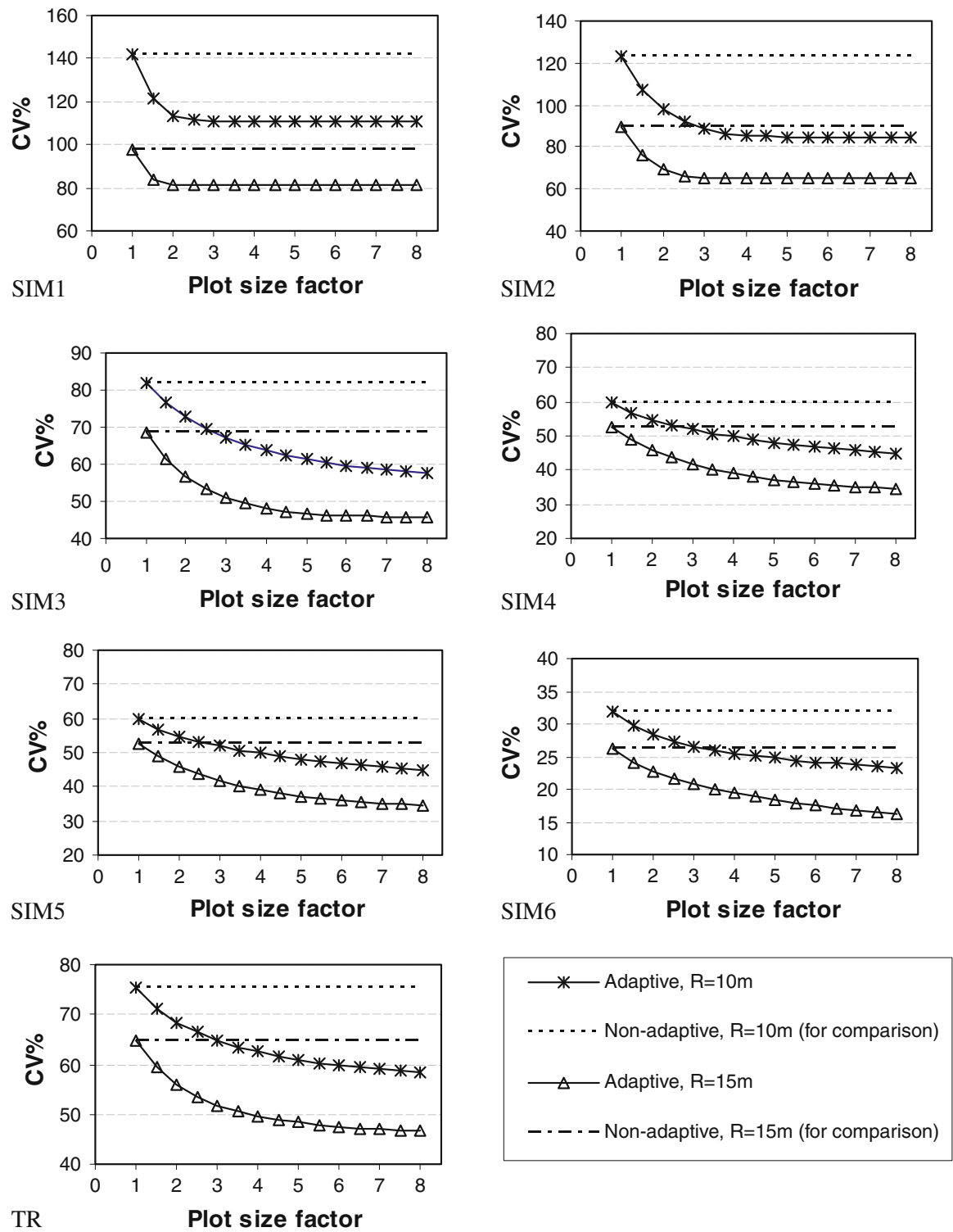

Fig. 5 Population coefficients of variation CV\% of the per-plot density observations as a function of PSF for the seven study populations. The CV\% values of simple random sampling are depicted as dotted and dashed horizontal line for the non-adaptive circular sample plots of 10 and $15 \mathrm{~m}$ radius, respectively

\subsection{Critical value}

Figure 6 illustrates the relationship between $\mathrm{CrV}$ and the statistical performance of the different plot designs for CrVs and PSFs: (1) the CV\% for the adaptive plots is less than the CV\% for nonadaptive plots (horizontal line in Fig. 6); and (2) the CV\% 
Table 1 Average percentage of expanded plots in the seven test populations

\begin{tabular}{|c|c|c|c|c|c|c|c|}
\hline \multirow[t]{2}{*}{$\mathrm{CrV}$} & \multicolumn{7}{|c|}{ Percentages of expanded plots } \\
\hline & $\begin{array}{l}\text { SIM1 } \\
\lambda=5 \mathrm{~m}\end{array}$ & $\begin{array}{l}\text { SIM2 } \\
\lambda=10 \mathrm{~m}\end{array}$ & $\begin{array}{l}\text { SIM3 } \\
\lambda=20 \mathrm{~m}\end{array}$ & $\begin{array}{l}\text { SIM4 } \\
\lambda=30 \mathrm{~m}\end{array}$ & $\begin{array}{l}\text { SIM5 } \\
\lambda=100 \mathrm{~m}\end{array}$ & $\begin{array}{l}\text { SIM6 } \\
\lambda=100 \mathrm{~m}\end{array}$ & $\begin{array}{l}\text { TR: mapped real } \\
\text { population of } \\
\text { Tamarix } \\
\text { ramosissim }\end{array}$ \\
\hline 1 & 7.13 & 10.72 & 19.65 & 31.00 & 83.25 & 81.70 & 24.10 \\
\hline 2 & 6.91 & 10.07 & 17.90 & 26.76 & 65.64 & 64.74 & 19.16 \\
\hline 3 & 6.74 & 9.52 & 16.56 & 23.67 & 48.61 & 50.90 & 16.83 \\
\hline
\end{tabular}

The initial plot radius is $15 \mathrm{~m}$

increases (slightly) with increasing $\mathrm{CrV}$. The latter rests with the fact that a $\mathrm{CrV}$ of 1 triggers the largest number of plots to be expanded. Table 1 gives the proportion of expanded plots for the different designs in Fig. 6.

The proportions of expanded plots depend on the spatial distribution of trees. SIM1 with its compact clusters has the lowest proportion of expanded plots. As well, the percentage of expanded clusters is smaller for larger CrVs. For population SIM1, for example, with $\mathrm{CrVs}=1,2$ or 3, the $\mathrm{CV} \%$ is pretty much constant across levels of PSF. An explanation is again in the spatial pattern: if an initial plot intersects a compact and dense cluster, there is likely more than one captured object which, everything else equal, tends to make results with $\mathrm{CrV}=1,2$ and 3 similar. For some populations, the effect of $\mathrm{CrV}$ is stronger and interacts with PSF. Examples of this are TR, SIM3 and SIM4 which exhibit fairly similar spatial patterns (c.f. Fig. 3).

The absolute gain in precision of estimated density from the adaptive plot design is highest for the compactly clustered populations SIM1 and SIM2, and lowest for the scattered clusters in population SIM5 and SIM6. For the statistical performance, the choice of PSF is generally much more critical than the choice of $\mathrm{CrV}$, the one exception is SIM1 with very compact clusters.

\section{Discussion and conclusions}

The comparison shows that the adaptive design is superior to sampling with the nonexpanded initial plots in all considered cases. Yet the relative performance depends (1) on the PSF, (2) on the CrV and above all (3) on the degree of clustering of the target objects in the study populations, as demonstrated in Fig. 6: for more widely scattered clusters, as in populations SIM5 and SIM6, the precision of sampling with nonadapted initial plots is only slightly less than for the adaptive plot design. However, in compactly clustered populations the adaptive design suggests a potential of sizeable improvements in precision without encumbering field work by complex, impractical and time-consuming plot-expansion rules as typically seen in conventional ACS designs (Smith et al. 2003; Su and Quinn II 2003; Turk and Borkowski 2005).

A conditional plot expansion also means that more time is needed for making additional field observations; time that could be equally devoted to a larger nonadaptive 

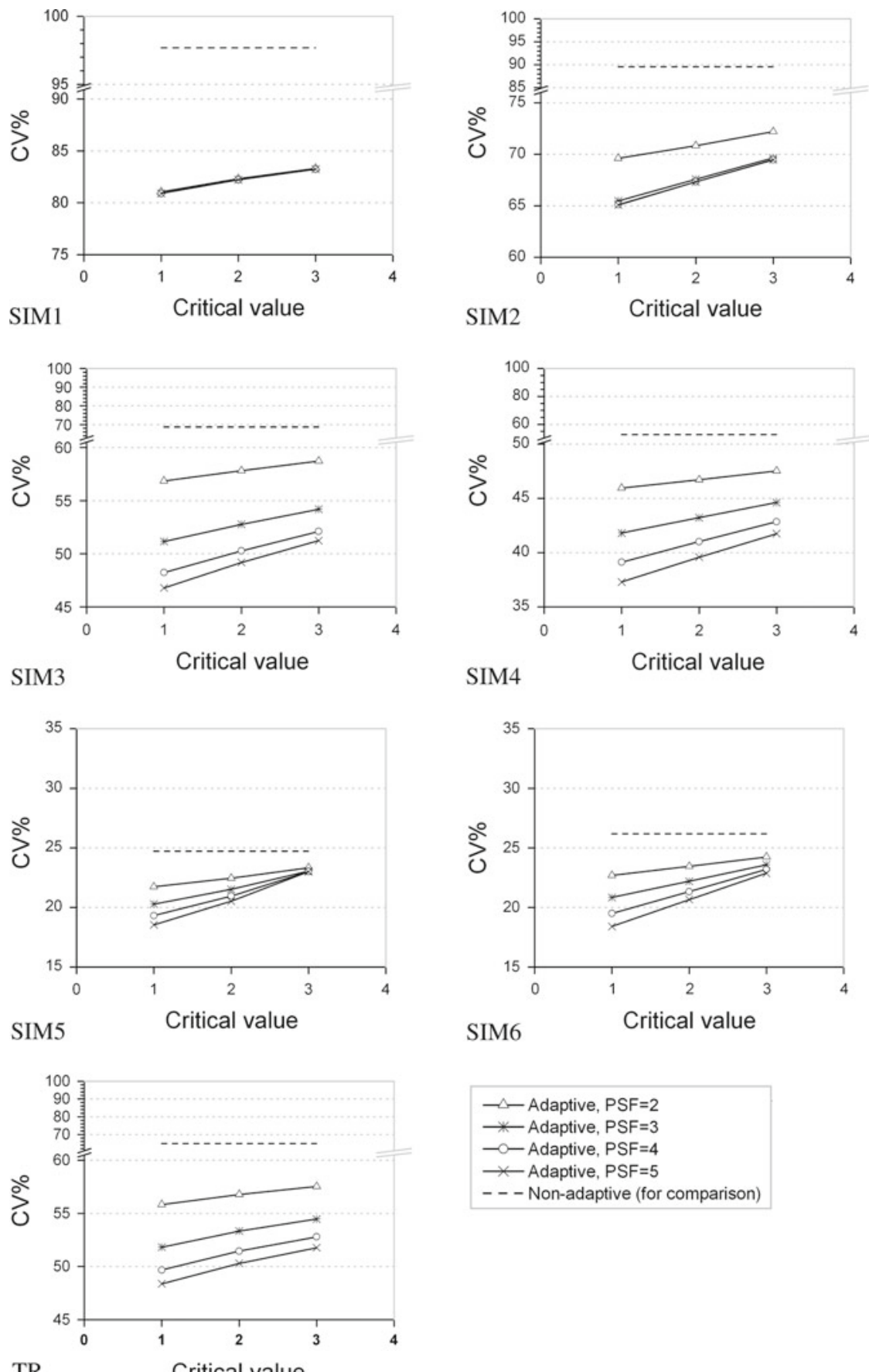

TR

Critical value

Fig. 6 Population coefficients of variation CV\% of the per-plot density observations for the seven study populations SIM1-SIM6 and TR for CrVs between 1 and 3 and PSFs from 2 to 5. Initial sample plot radius was $R=15 \mathrm{~m}$. For comparison, results for non-expanded initial plots are given as a horizontal dashed line 
sample size. However, in most forest inventories considerable time is spent for moving between sample locations. If a modest increase in the time spent on a single sample location is necessary to complete an expanded plot, the increase in total inventory time may not be a concern. The results obtained from our study only allow a rough estimation of the additional efforts: If determination of the coordinates of an object takes a fixed amount of time, and the number of objects to measure is proportional to the observed area, the additional time requirement can be obtained from the percentage of expanded plots (Table 1).

The number of expanded plots can be controlled, to a certain extent, by the choice of $\mathrm{CrV}$ : a higher $\mathrm{CrV}$ triggers fewer expansions (Table 1). Taking into account that the loss in precision by using a $\mathrm{CrV}$ of 2 or 3 is expected to be minor (Fig. 6) one might choose a $\mathrm{CrV}$ larger than 1 . Choosing a suitable $\mathrm{CrV}$ is also a strategic issue for the classic adaptive plot design (Brown 2003).

For any complex plot design that derives inclusion probabilities from inclusion zones (e.g. Kleinn and Vilčko 2006) the challenge is to find an efficient and fast numerical procedure that is geometrically correct. Determination of inclusion zones may, for example, be made easier by survey techniques that allow a rapid mapping of tree positions or models that can predict the inclusion zones from easy and rapidly observable features in a field plot. In this study we derived actual inclusion probabilities analytically in a GIS environment, so that field work is restricted to the determination of polar coordinates of relevant trees.

This study shares first experiences and insights to a new adaptive plot design with appeal to surveys of sparse yet spatially clustered populations. This method can be expected to be a viable alternative to standard ACS if it conserves most of the statistical efficiency attributed to ACS, as a cumbersome open-ended field work is excluded. It is obvious that overall efficiencies of this design and the standard ACS design vary from case to case, and thus a direct analytic comparison between them simply based on the variance estimator is impossible. Which approach is preferable depends on the actual conditions and cannot be answered in general.

Acknowledgments The results presented here are from the research project KL 894/10-1 funded by the German Science Council (DFG). We gratefully acknowledge this support from DFG. Our sincere thanks go to Dr. Frantisěk Vilčko who shared his skills and experiences in computer programming for determining inclusion zones, to Mr. Tim Exner for his kind assistance, to Mr. Paul Magdon for his comments on the graphic presentation of the study results, and Dr. Yuancai Lei and Mr. Guangyu Zhu for their support to field work when mapping our real population. Furthermore, we thank two anonymous reviewers for their helpful comments.

Open Access This article is distributed under the terms of the Creative Commons Attribution Noncommercial License which permits any noncommercial use, distribution, and reproduction in any medium, provided the original author(s) and source are credited.

\section{References}

Acharya B, Bhattarai G, Gier Ad, Stein A (2000) Systematic adaptive cluster sampling for the assessment of rare tree species in Nepal. For Ecol Manage 137:65-73

Acworth J (1999) Prunus africana. Striving for sustainable and equitable resource management in Cameroon. Med Plant Conserv 5:15-18 
Bih F (2007) Assessment methods for non-timber forest products in off-reserve forests: case study of Goaso district, Ghana. Dissertation, Universtiy of Freiburg

Brown JA (2003) Designing an efficient adaptive cluster sample. Environ Ecol Stat 10:95-105

Brown JA, Manly BJF (1998) Restricted adaptive cluster sampling. Environ Ecol Stat 5:49-63

Chao C-T, Thompson SK (1999) Incomplete adaptive cluster sampling designs. In: Proceedings of the section on survey research methods of the American statistical association, pp 345-350

Christman MC (1997) Efficiency of some sampling designs for spatially clustered populations. Environmetrics 8:145-166

Christman MC, Lan F (2001) Inverse adaptive cluster sampling. Biometrics 57:1096-1105

Diggle PJ (2003) Statistical analysis of spatial point patterns. Arnold, London

Dryver AL (1999) Adaptive sampling designs and associated estimators. Dissertation, The Pennsylvania State University, USA

Dryver AL (2003) Performance of adaptive cluster sampling estimators in a multivariate setting. Environ Ecol Stat 10:107-113

Dryver AL, Thompson SK (2005) Improved unbiased estimators in adaptive cluster sampling. J R Statist Soc B 67:157-166

Eriksson M (1995) Design-based approaches to horizontal-point-sampling. For Sci 41:890-907

Félix-Medina MH (2003) Asymptotics in adaptive cluster sampling. Environ Ecol Stat 10:61-82

Félix-Medina MH, Thompson SK (2004) Adaptive cluster double sampling. Biometrica 91:877-891

Gregoire TG, Valentine HT (2008) Sampling strategies for natural resources and the environment. Chapman \& Hall/CRC, Boca Raton

Hanselman DH, Quinn II TJ, Lunsford C, Heifetz J, Clausen D (2003) Applications in adaptive cluster sampling of Gulf of Alaska rockfish. Fish Bull 101:501-513

Kleinn C, Vilčko F (2006) A new empirical approach for estimation in $K$-tree sampling. For Ecol Manage 237:522-533

Lo NCH, Griffith D, Hunter JR (1997) Using a restricted adaptive cluster sampling to estimate pacific hake larval abundance. CalCOFI Rep 38:103-113

Magnussen S, Werner K, Leckie DG, Paradine D (2005) Adaptive cluster sampling for estimation of deforestation rates. Eur J For Res 207(220):207-220

Mandallaz D (2008) Sampling techniques for forest inventories. Chapman and Hall/CRC, Boca Raton

Muttlak HA, Khan A (2002) Adjusted two-stage adaptive cluster sampling. Environ Ecol Stat 9:111-120

Noon BR, Ishwar NM, Vasudevan K (2006) Efficiency of adaptive cluster and random sampling in detecting terrestrial herpetofauna in a tropical rainforest. Wildl Soc Bull 34:59-68

Phillipi T (2005) Adaptive cluster sampling for estimation of abundances within local populations of lowabundance plants. Ecology 86:1091-1100

Roesch FA Jr (1993) Adaptive cluster sampling for forest inventories. For Sci 39:655-669

Roesch FA Jr, Green EJ, Scott CT (1993) An alternative view of forest sampling. Stat Can 19:199-204

Salehi MM (1999) Rao-Blackwell versions of the Horvitz-Thompson and Hansen-Hurwitz in adaptive cluster sampling. Environ Ecol Stat 6:183-195

Salehi MM (2003) Comparison between Hansen-Hurwitz and Horvitz-Thompson estimators for adaptive cluster sampling. Environ Ecol Stat 10:115-127

Salehi MM, Seber GAF (1997) Adaptive cluster sampling with networks selected without replacement. Biometrica 84:209-219

Salehi MM, Seber GAF (2002) Unbiased estimators for restricted adaptive cluster sampling. Aus N Z J Stat 44:63-74

Smith DR, Villella RF, Lemarié DP (2003) Application of adaptive cluster sampling to low-density populations of freshwater mussels. Environ Ecol Stat 10:7-15

Su Z, Quinn II TJ (2003) Estimator bias and efficiency for adaptive cluster sampling with order statistics and a stopping rule. Environ Ecol Stat 10:17-41

Talvitie M, Leino O, Holopainen M (2006) Inventory of sparse forest populations using adaptive cluster sampling. Silva Fennica 40:101-108

Thompson SK (1990) Adaptive cluster sampling. J Am Stat Assoc 85:1050-1059

Thompson SK (1991) Stratified adaptive cluster sampling. Biometrica 78:389-397

Thompson WL (2004) Sampling rare or elusive species: concept, designs, and techniques for estimating population parameters. Island Press, Washington

Turk P, Borkowski JJ (2005) A review of adaptive cluster sampling: 1990-2003. Environ Ecol Stat 12:55-94 
Valentine HT, Gove JH, Gregoire TG (2001) Monte Carlo approaches to sampling forested tracts with lines or points. Can J For Res 31:1410-1424

Wong J (2001) Biometrics and NTFP inventory. In: Biometry, modelling and information science conference, University of Greenwich, London, England

\section{Author Biographies}

Haijun Yang is a doctoral student at the Chair of forest inventory and remote Sensing at Georg-AugustUniversität Göttingen, Germany. His current research is focused on adaptive sampling for forest inventory and ecological surveys.

Christoph Kleinn is a professor of forest inventory and remote sensing, and the director of Center for Tropical and Subtropical Agriculture and Forestry, Georg August University in Gottingen, Germany.

Lutz Fehrmann is a postdoc at the Chair of forest inventory and remote Sensing at Georg-August-Universität Göttingen, Germany. His current research covers sampling techniques and biomass modeling.

Shouzheng Tang is a professor of forest management at the Research Institute of Forest Resource Information Techniques, Chinese Academy of Forestry, and an academician of the Chinese Academy of Sciences.

Steen Magnussen is a senior research scientist of forest inventory and analysis at Canadian Forest Service. 